\title{
Colistin and polymyxin B for treatment of nosocomial infections in intensive care unit patients: pharmacoeconomic analysis
}

\author{
Júlia Coelho França Quintanilha ${ }^{1}$. Natalia da Costa Duarte ${ }^{1}$. Gustavo Rafaini Lloret ${ }^{1}$. Marília Berlofa Visacri ${ }^{1}$. \\ Karen Prado Herzer Mattos ${ }^{1}$. Desanka Dragosavac ${ }^{1} \cdot$ Antonio Luis Eiras Falcão $^{1} \cdot$ Patricia Moriel $^{2}$
}

Received: 21 June 2018 / Accepted: 6 December 2018 / Published online: 15 December 2018

(c) Springer Nature Switzerland AG 2018

\begin{abstract}
Background The emergence and rapid spread of multidrug-resistant gram-negative bacteria related to nosocomial infections is a growing worldwide problem, and polymyxins have become important due to the lack of new antibiotics. Objectives To evaluate the outcomes and pharmacoeconomic impact of using colistin and polymyxin B to treat nosocomial infections. Setting Neurosurgical, cardiovascular, or transplantation intensive care unit (ICU) at the Clinical Hospital of the University of Campinas (São Paulo, Brazil). Method A retrospective cohort study was conduct in patients in the ICU. The renal function was determined daily during treatment by measuring the serum creatinine. A cost minimization analysis was performed to compare the relative costs of treatment with colistin and polymyxin B. Main outcomes measure The outcomes were 30-day mortality and frequency and onset of nephrotoxicity after beginning treatment. Results Fifty-one patients treated with colistin and 51 with polymyxin B were included. 30-day mortality was observed in $25.49 \%$ and $33.33 \%$ of patients treated with colistin and polymyxin B, respectively; Nephrotoxicity was observed in $43.14 \%$ and $54.90 \%$ of patients in colistin and polymyxin B groups, respectively; and onset time of nephrotoxicity was $9.86 \pm 13.22$ days for colistin and $10.68 \pm 9.93$ days for polymyxin B group. Colistin treatment had a lower cost per patient compared to the cost for polymyxin B treatment (USD \$13,389.37 vs. USD \$13,639.16, respectively). Conclusion We found no difference between 30-day mortality and nephrotoxicity between groups; however, colistin proved to be the best option from a pharmacoeconomic point of view.
\end{abstract}

Keywords Brazil $\cdot$ Colistin $\cdot$ Hospital infection $\cdot$ Intensive care $\cdot$ Multidrug-resistant bacteria $\cdot$ Nephrotoxicity $\cdot$ Pharmacoeconomics $\cdot$ Polymyxin B

\section{Impacts on practice}

- Nephrotoxicity and 30-day mortality are frequent in patients treated with colistin and polymyxin B.

Júlia Coelho França Quintanilha and Natalia da Costa Duarte have contributed equally to this study.

Patricia Moriel

patricia.moriel@fcf.unicamp.br

1 School of Medical Sciences, University of Campinas (UNICAMP), Tessália Vieira de Camargo 126, 13083-887 Campinas, SP, Brazil

2 Faculty of Pharmaceutical Sciences, University of Campinas (UNICAMP), Cândido Portinari 200, 13083-871 Campinas, SP, Brazil
- The pharmacoeconomic analysis supports the choice of the best treatment for patients in intensive unit care with multidrug-resistant infections.

\section{Introduction}

The emergence and rapid spread of multidrug-resistant (MDR) gram-negative bacteria related to nosocomial infections is a growing worldwide problem. Infections caused by pathogens, such as Acinetobacter baumannii and Pseudomonas aeruginosa, are often statistically significantly related to increased length of intensive care unit (ICU) and hospital stay, as well as high mortality in patients cared for in the ICU [1-3]. Polymyxins have become important due to the lack of new antibiotics and limited development of new agents [2, 4]. In many cases, 
pathogens causing nosocomial infections are resistant to most of the currently available antibiotics [5].

Although polymyxins are being administered more often, their clinical use has been restricted by limited pharmacokinetic, pharmacodynamic, and toxicodynamic data, because they were never subjected to the drug development process required for compliance with contemporary regulatory requirements when they were introduced in the 1960s [6, 7]. This may have led to increasing resistance to polymyxins, possibly due to the use of suboptimal dosing regimens arising from an insufficient understanding of the pharmacokinetics and pharmacodynamics of these drugs [5].

The use of polymyxins may lead to a significant risk of developing nephrotoxicity, the main adverse reaction to these drugs, with the incidence of acute renal injury varying between 20 and 60\% [8-10]. Nephrotoxicity may be associated with a worse prognosis for the patients, since they are vulnerable and infected with MDR bacteria [11]. Therefore, it is critical to determine whether polymyxin B and colistin have different nephrotoxicity profiles in a clinical setting.

Clinicians, pharmacists, and laboratorians all struggle with how to best use polymyxins in practice. On the one hand, polymyxins are viewed as last resort drugs for treating patients with infections caused by MDR gram-negative bacteria. On the other hand, there are global challenges associated with their use because of their toxicity, the lack of reliable testing methods, and difficulty in establishing breakpoints. According to the Clinical Laboratory Standards Institute (CLSI)/European Committee on Antimicrobial Susceptibility Testing (EUCAST) Joint Working Group, the recommended breakpoint of polymyxins for resistant Acinetobacter spp. and Pseudomonas aeruginosa is $\geq 4 \mu \mathrm{g} / \mathrm{ml}$ [12].

Colistin dosing is undoubtedly more complicated than polymyxin $\mathrm{B}$ dosing due to the complex interplay between the pro-drug and active moiety, the differing package insert recommendations in various parts of the world, and the confusion among dosing units [13]. In patients with normal renal function, there are three major approaches to colistin dosing: that of the US package insert, the European Medicines Agency (EMA) package insert, and dosing per the pharmacokinetic equation set forth by Garonzik et al. [14]. Therefore, polymyxin dosing recommendations differ between polymyxin B and colistin and vary significantly (e.g. from $1 \mathrm{mg} / \mathrm{kg} /$ day to $5 \mathrm{mg} / \mathrm{kg} /$ day according to creatinine clearance) [15].

There is an association between the development of bacterial resistance and increased cost [16]. Moreover, druginduced complications can also result in substantial economic burdens to institutions. Either of these problems can increase length of stay, which is the single most important variable influencing the overall cost of patient care [13].
Therefore, new clinical and economic data on polymyxins are required.

\section{Aim of the study}

We aimed to evaluate the outcomes (30-day mortality and nephrotoxicity) and pharmacoeconomic impact of using colistin and polymyxin B to treat nosocomial infections caused by gram-negative bacteria in patients in the ICU.

\section{Ethics approval}

The study was approved by the Institutional Research Ethics Board of Cinical Hospital of Medical Sciences, University of Campinas (16330513.8.0000.5404 and 24219414.1.0000.5404).

\section{Method}

\section{Study design}

We performed a retrospective cohort study at the Clinical Hospital of the University of Campinas (São Paulo, Brazil) during a 3-year period (August 2010-July 2013). Patients who were $>18$ years old, admitted to a neurosurgical, cardiovascular, or transplantation ICU and who were administered colistin or polymyxin B for at least $72 \mathrm{~h}$ for a clinically diagnosed and microbiologically documented nosocomial infection caused by gram-negative bacteria were eligible for inclusion. Only polymyxin B was available at the hospital during the first 18 months of the study, and only colistin was available during the last 18 months. Thus, only the first episode of infection of each patient treated with one of the polymyxins was considered in this analysis. We excluded patients who did not undergo an antimicrobial susceptibility test and for whom polymyxin was empirically indicated.

\section{Microbiological assays}

Antimicrobial susceptibility testing was performed according to the CLSI guidelines (formerly the National Committee for Clinical Laboratory Standards) [17] in the Clinical Microbiology Laboratory of the Clinical Hospital at the University of Campinas. Routine laboratory susceptibility testing procedures for commonly used antibiotics were performed using an automated broth microdilution method (Vitek 2; bioMérieux, Hazelwood, MO, USA). An isolate with a minimum inhibitory polymyxin concentration of $\leq 2 \mathrm{~g} / \mathrm{mL}$ was considered susceptible when testing with this system [16]. The acquired resistance profile for each 
bacteria strain that was isolated was classified according to the European Society of Clinical Microbiology and Infectious Diseases [18].

\section{Data extraction}

At the date of admission into the ICU, illness severity was evaluated according to the acute physiological and chronic health evaluation II (APACHE II) score and the sequential organ failure assessment (SOFA). Sex, age, presence of diabetes mellitus, length of ICU stay, and complications during the stay (tracheostomy, mechanical ventilation, pneumonia, ventilator-associated pneumonia, sepsis, septic shock, and urinary tract infection) were also evaluated. Further, clinical samples isolated from the infection site, daily serum creatinine level, and polymyxin dose and duration of therapy were noted and all these parameters described were evaluated as factors potentially associated with the clinical outcomes defined below.

\section{Clinical outcomes}

The outcomes were 30-day mortality (mortality in 30 days due to any cause, including drug-related toxicities, the infection itself, or any other type of complication during treatment) and frequency and onset of nephrotoxicity after beginning polymyxin treatment. The renal function was determined daily during treatment by measuring the serum creatinine. Renal impairment was defined as a creatinine increase of $\geq 0.3 \mathrm{mg} / \mathrm{dL}$ or $\geq 1.5$-fold from the baseline level in accordance with Stage 1 of the Acute Kidney Injury (AKIN) criteria $[19,20]$.

\section{Cost analysis}

A cost minimization analysis was performed to compare the relative costs of treatment with colistin and polymyxin B. The cost evaluation included expected antibiotic cost (polymyxin B and colistin) and ICU hospitalization costs. Costs were expressed in 2015 US dollars (1 USD $\$=\mathrm{R} \$$ 3.75 Brazilian real). The values were adjusted to September 2018 (13\%) by the annual inflation index of Brazil-IPCA [21]. The costs of polymyxin B ampules of 500,000 IU and colistin ampule bottles of 1,000,000 IU were USD \$15.04 and USD \$4.01, respectively, according to the current purchase price from the Institution and in accordance with the Brazilian Drug Market Regulation Chamber (CMED in Portuguese) [22]. The cost for ICU hospitalization per day is USD \$356.85 according to the DATASUS database [23]. A univariate sensitivity analysis was performed to evaluate the impact of the parameters (antibiotic and ICU hospitalization) on the cost of treatment.

\section{Data analysis}

Statistical analyses were performed with the SAS System for Windows 9.4 program (SAS Institute, Cary, NC, USA), and data were analyzed using the Chi square, Fisher's exact, and Mann-Whitney tests. A two-tailed $P$ value of $\leq 0.05$ was considered significant for all tests.

\section{Results}

\section{Clinical and demographic characteristics}

The study screened 190 patients that fulfilled the inclusion criteria. Eighty-eight patients were excluded as they did not have the susceptibility test performed prior to polymyxin treatment. A total of 102 patients were included in the study; 51 were treated with polymyxin $B$ and 51 were treated with colistin. The clinical and demographic characteristics of the patients are summarized in Table 1.

\section{Antimicrobial resistance analysis}

Tracheal secretion was the most frequent site for isolating bacterial strains in both groups, followed by blood (Table 1). A. baumannii and $P$. aeruginosa were the most frequently isolated pathogens, accounting for $63.7 \%$ and $7.8 \%$ of isolates, respectively. Antibiotic resistance was observed; $A$. baumannii showed alarming resistance to almost all antibiotics tested but remained susceptible to polymyxins with optimal sensitivity (Table 2).

\section{Outcomes}

Considering both groups of patients treated with colistin and polymyxin B, 30-day mortality was observed in $29.41 \%$ of subjects and nephrotoxicity in $49.01 \%$. However, these parameters and onset time of nephrotoxicity was not statistically different between the groups (Table 3 ). The resolution of nephrotoxicity was more frequent in the polymyxin B group (11 patients, $39.39 \%$ ) than in the colistin group (6 patients, $27.27 \%$ ); however, the patients treated with colistin group took an average of $11.50 \pm 5.82$ days to resolve nephrotoxicity and the polymyxin B group of patients took $17.27 \pm 20.16$ days.

Regarding the pharmacoeconomic analysis, since colistin and polymyxin B were found to show no statistical difference regarding nephrotoxicity and 30-day mortality, a cost minimization analysis was performed to compare the cost difference between groups. We observed that colistin treatment had a lower cost per patient compared to the cost for polymyxin B treatment (USD $\$ 13,389.37$ vs. USD $\$ 13,639.16$, respectively) (Table 4). 
Table 1 Demographic and clinical characteristics

\begin{tabular}{|c|c|c|c|}
\hline & $\begin{array}{l}\text { Colistin }(\mathrm{n}=51) \\
{[\text { mean } \pm \mathrm{SD} \text { or } \mathrm{n}(\%)]}\end{array}$ & Polymyxin B (n=51) & $p$-value \\
\hline \multicolumn{4}{|l|}{ Demographic } \\
\hline Age (years) & $49.71 \pm 18.14$ & $53.16 \pm 15.20$ & $0.4140^{\mathrm{a}}$ \\
\hline Sex (male) & $42(82.35 \%)$ & $35(68.63 \%)$ & $0.1071^{\mathrm{b}}$ \\
\hline APACHE II score & $15.3 \pm 5.6$ & $15.9 \pm 5.4$ & $0.7149^{\mathrm{a}}$ \\
\hline SOFA score & $7.2 \pm 2.8$ & $6.9 \pm 2.8$ & $0.5976^{\mathrm{a}}$ \\
\hline \multicolumn{4}{|l|}{ Comorbidities } \\
\hline Diabetes mellitus & $8(15.7)$ & $10(19.6)$ & $0.5711^{\mathrm{b}}$ \\
\hline \multicolumn{4}{|l|}{ Complications during ICU stay } \\
\hline Tracheostomy & $23(43.4)$ & $27(48.2)$ & $0.6138^{\mathrm{b}}$ \\
\hline Mechnical ventilation (MV) & $49(92.5)$ & $49(87.5)$ & $0.3908^{\mathrm{b}}$ \\
\hline MV $>7$ days & $42(79.2)$ & $45(80.4)$ & $0.3370^{\mathrm{b}}$ \\
\hline Pneumonia & $16(30.2)$ & $24(42.9)$ & $0.1702^{\mathrm{b}}$ \\
\hline Ventilator-associated pneumonia & $9(17.0)$ & $14(25.0)$ & $0.3051^{\mathrm{b}}$ \\
\hline Sepsis & $16(30.2)$ & $26(46.4)$ & $0.0816^{\mathrm{b}}$ \\
\hline Septic shock & $10(18.9)$ & $18(32.1)$ & $0.1128^{\mathrm{b}}$ \\
\hline Urinary tract infection & $1(1.9)$ & $7(12.5)$ & $\mathbf{0 . 0 3 3 6} 6^{b}$ \\
\hline Clinical samples isolated & 51 & 51 & NA \\
\hline \multicolumn{4}{|l|}{ Infection site } \\
\hline Tracheal Secretion & $35(68.6)$ & $37(72.6)$ & $0.5423^{b}$ \\
\hline Blood & $9(17.6)$ & $9(17.6)$ & $0.9040^{\mathrm{b}}$ \\
\hline Urinary tract & $1(2.0)$ & $1(2.0)$ & $0.5910^{\mathrm{b}}$ \\
\hline Other & $6(11.8)$ & $4(7.8)$ & $0.2091^{\mathrm{b}}$ \\
\hline \multicolumn{4}{|l|}{ Treatment } \\
\hline Duration of therapy (days) & $9.9 \pm 4.8$ & $11.5 \pm 4.9$ & $0.1121^{\mathrm{a}}$ \\
\hline Average daily dose ( $\left.10^{6} \mathrm{IU}\right)$ & $3.3 \pm 1.5$ & $1.5 \pm 0.4$ & NA \\
\hline Total cumulative dose $\left(10^{6} \mathrm{IU}\right)$ & $32.3 \pm 2.4$ & $16.7 \pm 9.0$ & NA \\
\hline
\end{tabular}

The words are in bold because they are different topics

$I C U$ intensive care unit, $I U$ international Unit, $N A$ not applied, $n$ number of subjects, $S D$ standard deviation Statistical analysis: ${ }^{\mathrm{a}} \mathrm{Mann}-\mathrm{Whitney}$ test; ${ }^{\mathrm{b}} \mathrm{Chi}$ square test or Fisher's exact test
Colistin treatment was about USD \$249.79 (1.8\%) cheaper than polymyxin B treatment. Considering the univariate sensitivity analysis, the cost minimization results favored treatment with colistin regarding antibiotic and total cost (Table 4).

\section{Discussion}

Polymyxins have gained attention as an alternative for the lack of new and effective treatment options for infections caused by MDR Gram-negative bacteria, becoming the mainstay of treatment [24]. Nevertheless, the use of these agents is restricted because there are limited data on their efficacy and potential toxicity.

Both colistin and polymyxin B are generated from Bacillus polymyxa through a fermentation process [25]. Polymyxin B is administered as the active drug, whereas colistin is administered as the pro-drug, colistimethate sodium
(CMS). When colistin is formulated as a pro-drug, it is subjected to further manufacturing processes such as sulfomethylation and hydrolysis that can contribute to variations in toxicity and efficacy of this antimicrobial [26].

Originally, polymyxin B was thought to be more potent and more nephrotoxic than colistin [27]. However, this subject remains unclear since newer reports showed that both drugs have minor differences in antimicrobial activities with no significant difference in mortality [28]. Additionally, colistin has been associated with more episodes of nephrotoxicity [28]. In particular, rates of nephrotoxicity vary substantially as new data are published describing both drugs, mainly because the majority of older reports did not provide a formal definition of nephrotoxicity [29]. In our study, patients treated with polymyxin B showed a higher frequency of nephrotoxicity compared to those treated with colistin (54.90\% and $43.14 \%$ respectively); however, despite being clinically relevant, this difference was not statistically significant. These rates differ from the nephrotoxicity rates 
Table 2 Antimicrobial resistance of A. baumannii and P. aeruginosa isolates

\begin{tabular}{lll}
\hline Antibiotic & \multicolumn{2}{l}{$\begin{array}{l}\text { Number of resistant isolates/total isolates } \\
\text { tested }(\%)\end{array}$} \\
\cline { 2 - 3 } & A. baumannii $(\mathrm{n}=65)$ & P. aeruginosa $(\mathrm{n}=8)$ \\
\hline Imipenem & $64 / 64(100.0)$ & $8 / 8(100.0)$ \\
Meropenem & $61 / 61(100.0)$ & $8 / 8(100.0)$ \\
Ciprofloxacin & $52 / 52(100.0)$ & $7 / 7(100.0)$ \\
Cefepime & $62 / 62(100.0)$ & $8 / 8(100.0)$ \\
Ceftazidime & $61 / 61(100.0)$ & $8 / 8(100.0)$ \\
Piperacillin/Tazobac- & $41 / 41(100.0)$ & $6 / 8(75.0)$ \\
tam & & \\
Ampicillin/Sulbactam & $50 / 60(86.7)$ & $1 / 2(50.0)$ \\
Levofloxacin & $9 / 9(100.0)$ & $2 / 2(100.0)$ \\
Gentamicin & $42 / 63(66.7)$ & $5 / 8(62.5)$ \\
Amikacin & $51 / 61(80.4)$ & $3 / 8(37.5)$ \\
Polymyxin B & $\mathbf{1 / 3 5}(\mathbf{2 . 8})$ & $\mathbf{0 / 3}(\mathbf{0 . 0})$ \\
Colistin & $\mathbf{0 / 2 8}(\mathbf{0 . 0})$ & $\mathbf{0 / 5}(\mathbf{0 . 0})$ \\
\hline
\end{tabular}

The words are in bold because they are the main topic of this study $n$ number of subjects

Table 3 Outcomes of colistin and polymyxin B treatment Colistin $(\mathrm{n}=51) \quad$ Polymyxin B $(\mathrm{n}=51) \quad p$-value $[\mathrm{n}(\%)$ or mean $\pm \mathrm{SD}$ ]

\begin{tabular}{llll}
\hline 30-day mortality & $13(25.49)$ & $17(33.33)$ & $0.3847^{\mathrm{a}}$ \\
Nephrotoxicity & $22(43.14)$ & $28(54.90)$ & $0.2347^{\mathrm{a}}$ \\
Onset time $^{\mathrm{c}}$ (days) & $9.86 \pm 13.22$ & $10.68 \pm 9.93$ & $0.2432^{\mathrm{b}}$ \\
\hline
\end{tabular}

$n$ number of subjects, $S D$ standard deviation

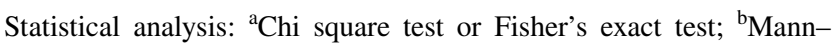
Whitney test; ' Onset time: length between start treatment and toxicity manifestation published in a recent meta-analysis $(21.0-46.0 \%$ in polymyxin B treated patients and $24.0-74.0 \%$ in colistin-treated patients) [27]. These rates are also higher comparable to those published by Oliveira et al. [30] who evaluated the difference in the rates of renal impairment between these agents and found no difference between them $(26.0 \%$ and $27.0 \%$ of patients in colistin and polymyxin groups, respectively; $p=0.92$ ). Additionally, a report by Phe et al. [8] showed that overall nephrotoxicity was found in 24 intention-totreat patients $(23.1 \%)$ who received polymyxin B versus 41 (33.9\%) who received colistimethate $(p=0.08)$ even though APACHE II score was not assessed in those patients.

The resolution of nephrotoxicity is also an important point to observe regarding polymyxin toxicity. We observed that nephrotoxicity resolved in more of those treated with polymyxin B compared to the colistin group (39.39\% and $27.27 \%$ respectively). However, patients treated with colistin showed a lower average number of days to resolution compared to the polymyxin B group $(11.50 \pm 5.82$ and $17.27 \pm 20.16$ respectively).

The results showed no difference in 30-day mortality between the colistin and polymyxin B group and it is comparable to Rigatto et al. [31] multicenter cohort study in which the overall 30-day mortality was $30.9 \%$ (25 patients) and $42.0 \%$ (178 patients) in the CMS and polymyxin B groups, respectively $(p=0.083)$. However, important variables such as another antimicrobial and time to appropriate antibiotic treatment were not evaluated and these limitations require further investigation regarding mortality.

Nephrotoxicity is the most common adverse effect associated with polymyxins use [2] and is a major concern. The total cumulative CMS dose has been associated with kidney damage, suggesting that shortening the treatment duration could decrease the incidence of kidney damage [2].
Table 4 Sensitivity analysis of colistin and polymyxin B treatment

\begin{tabular}{lccc}
\hline & $\begin{array}{l}\text { Colistin }(\mathrm{n}=51) \\
\text { Mean costs/patient }\end{array}$ & $\begin{array}{c}\text { Polymyxin B }(\mathrm{n}=51) \\
\text { (USD })\end{array}$ & $\begin{array}{l}\Delta \text { Costs: Colistin- } \\
\text { Polymyxin B (USD \$) }\end{array}$ \\
\hline ICU hospitalization & $\mathbf{1 3 2 5 9 . 4 2}$ & $\mathbf{1 3 1 1 9 . 0 8}$ & $\mathbf{+ 1 4 0 . 3 4}$ \\
$+20 \%$ & 15911.30 & 15742.90 & +168.40 \\
$-20 \%$ & 10607.54 & 10495.27 & +112.27 \\
Antibiotic & $\mathbf{1 2 9 . 9 5}$ & $\mathbf{5 2 0 . 0 8}$ & $\mathbf{- 3 9 0 . 1 3}$ \\
$+20 \%$ & 155.94 & 624.10 & -468.16 \\
$-20 \%$ & 103.96 & 416.06 & -312.10 \\
Total cost & $\mathbf{1 3 3 8 9 . 3 7}$ & $\mathbf{1 3 6 3 9 . 1 6}$ & $-\mathbf{2 4 9 . 7 9}$ \\
$+20 \%$ & 16067.24 & 16366.99 & -299.75 \\
$-20 \%$ & 10711.50 & 10911.33 & -199.83 \\
\hline
\end{tabular}

The words in bold represent different topics $n$ number of subjects, USD United States dollar

Statistical analysis: ${ }^{a}$ Chi square test or Fisher's exact test; ${ }^{b}$ Mann-Whitney test; ${ }^{\mathrm{c}}$ Onset time: length between start treatment and toxicity manifestation 
Polymyxin B and colistin have similar chemical structure [32]. Polymyxin B is minimally affected by kidney function [26]. Colistin is mainly cleared by non-renal mechanisms that are not fully understood, and only a small part is cleared by the kidneys [7]. It is therefore expected that following its administration, a higher level of CMS should be observed in the kidneys compared to renal level of polymyxin B after polymyxin B administration. Because of this we expected that renal impairment would be more quickly observed (through an increase in serum creatinine) in the colistin group. However, no significant difference was observed between groups based on this parameter.

To the best of our knowledge, this is the first study to perform a pharmacoeconomic analysis comparing the difference between colistin and polymyxin B treatment. Our cost minimization analysis shows that treatment with colistin costs less per patient compared to using polymyxin $\mathrm{B}$. This result may vary depending on the hospital, since antibiotic and ICU hospitalization costs differ. However, after considering a $20 \%$ difference, the univariate sensitivity analysis showed the economic advantage of colistin remained with respect to the total cost.

Limitations associated with retrospective studies should be considered. Furthermore, the data were collected initially for clinical rather than research purposes; thus, some information may have been absent, incomplete, or missing, such as other concomitant medications and death causes. Some cases of bacterial colonization may have been treated as clinical infections, and it was not possible to evaluate the concomitant use of other antibiotics. We could not evaluate costs associated with adverse events during antibiotic treatment.

Our study encourages future studies in this subject, mainly those ones evaluating complementary variables, such as other medications, concomitant infections, other complications from treatment, and try to associate mortality with these other variables as well.

\section{Conclusion}

In conclusion, we found no significant difference between 30-day mortality and nephrotoxicity between colistin and polymyxin $\mathrm{B}$ for the treating nosocomial infections in ICU patients. We also showed, for the first time, a cost advantage of using colistin over polymyxin B treatment. This can be important when deciding which antimicrobial to use for treating MDR gram-negative bacterial infections.

Acknowledgements The authors would like to thank the statistical office of School of Medical Sciences of University of Campinas (UNICAMP) for performing this statistical analysis.
Funding This work was supported by Conselho Nacional de Desenvolvimento Científico e Tecnológico $(\mathrm{CNPq})$ and Coordenação de Aperfeiçoamento de Pessoal de Nível Superior (CAPES) (Grant Numbers $164796 / 2014-2$ and $02 P 4353 / 2015$ to G.F.L).

Conflicts of interest The Authors declare no conflict of interest.

\section{References}

1. Barnett AG, Page K, Campbell M, Martin E, Rashleigh-Rolls R, Halton $\mathrm{K}$, et al. The increased risks of death and extra lengths of hospital and ICU stay from hospital-acquired bloodstream infections: a case-control study. BMJ Open. 2013;3:e003587.

2. Biswas S, Brunel JM, Dubus JC, Reynaud-Gaubert M, Rolain JM. Colistin: an update on the antibiotic of the 21 st century. Expert Rev Anti Infect Ther. 2012;10:917-34.

3. Michalopoulos AS, Karatza DC. Multidrug-resistant Gramnegative infections: the use of colistin. Expert Rev Anti Infect. 2010;8:1009-17.

4. Vincent JL, Rello J, Marshall J, Silva E, Anzueto A, Martin CD, et al. International study of the prevalence and outcomes of infection in intensive care units. JAMA. 2009;302:2323-9.

5. Zavascki AP, Goldani LZ, Cao G, Superti SV, Lutz L, Barth AL, et al. Pharmacokinetics of intravenous polymyxin B in critically ill patients. Clin Infect Dis. 2008;47:1298-304.

6. Li J, Nation RL, Milne RW, Turnidge JD, Coulthard K. Evaluation of colistin as an agent against multi-resistant gram-negative bacteria. Int J Antimicrob Agents. 2005;25:11-25.

7. Li J, Nation RL, Turnidge JD, Milne RW, Coulthard K, Rayner $\mathrm{CR}$, et al. Colistin: the re-emerging antibiotic for multidrugresistant gram-negative bacterial infections. Lancet Infect Dis. 2006;6:589-601.

8. Phe K, Lee Y, McDaneld PM, Prasad N, Yin T, Figueroa DA, Musick WL, Cottreau JM, Hu M, Tam VH. In vitro assessment and multicentre cohort study of comparative nephrotoxicity rates associated with colistimethate versus Polymyxin B therapy. Antimicrob Agents Chemother. 2014;58:2740-6.

9. Akajagbor DS, Wilson SL, Shere-Wolfe KD, Dakum P, Charurat ME, Gilliam BL. Higher incidence of acute kidney injury with intravenous colistimethate sodium compared with Polymyxin B in critically ill patients at a tertiary care medical center. Clin Infect Dis. 2013;57:1300-3.

10. Tuon FF, Rigatto MH, Lopes CK, Kamei LK, Rocha JL, Zavascki AP. Risk factors for acute kidney injury in patients treated with polymyxin B or colistin methanesulfonate sodium. Int J Antimicrob Agents. 2014;43:349-52.

11. Rigatto MH, Behle TF, Falci DR, Freitas T, Lopes NT, Nunes M, Costa LW, Zavascki AP. Risk factors for acute kidney injury (AKI) in patients treated with polymyxin B and influence of AKI on mortality: a multicentre prospective cohort study. J Antimicrob Chemother. 2015;70:1552-7.

12. CLSI Subcommittee On Antimicrobial Susceptibility Testing CLSI AST News Update. 2016. https://clsi.org/media/1700/clsinews-winter-2016.pdf. Accessed Sept 2018.

13. Birmingham MC, Hassett JM, Schentag JJ, Paladino JA. Assessing antibacterial pharmacoeconomics in the intensive care unit. Pharmacoeconomics. 1997;12:637-47.

14. Garonzik SM, Li J, Thamlikitkul V, Paterson DL, Shoham S, Jacob J, et al. Population pharmacokinetics of colistin methanesulfonate and formed colistin in critically ill patients from a multicenter study provide dosing suggestions for various categories of patients. Antimicrob Agents Chemother. 2011;55:3284-94. 
15. Pogue JM, Ortwine JK, Kaye KS. Clinical considerations for optimal use of the polymyxins: a focus on agent selection and dosing. Clin Microbiol Infect. 2017;23:229-33.

16. Allegranzi B, Nejad SB, Combescure C, Graafmans W, Attar H, Donaldson L, et al. Burden of endemic health-care-associated infection in developing countries: systematic review and metaanalysis. Lancet. 2011;377:228-41.

17. Clinical and Laboratory Standards Institute (CLSI). Performance standards for antimicrobial susceptibility testing. 23th informational supplement M100-S23 2013; Wayne, CLSI.

18. Magiorakos AP, Srinivasan A, Carey RB, Carmeli Y, Falagas ME, Giske CG, et al. Multidrug-resistant, extensively drug-resistant and pandrug-resistant bacteria: an international expert proposal for interim standard definitions for acquired resistance. Clin Microbiol Infect. 2012;18:268-81.

19. Leung KCW, Tonelli M, James MT. Chronic kidney disease following acute kidney injury-risk and outcomes. Nat Rev Nephrol. 2013;9:77-85.

20. National Kidney Foundation. K/DOQI clinical practice guidelines for chronic kidney disease: evaluation, classification, and stratification. Am J Kidney Dis. 2002;39:S1-266.

21. Citizen's Calculator-Central Bank of Brazil. 2018. https:// www3.bcb.gov.br/CALCIDADAO/publico/exibirFormCorre caoValores.do?method=exibirFormCorrecaoValores. Accessed Oct 2018.

22. Brazilian Drugs Market Regulation Chamber (CMED). Products price list, January 2015.

23. Brazilian Unified Health System (SUS). Price table, January 2015.
24. Gomes EC, Falci DR, Bergo P, Zavascki AP, Rigatto MH. Impact of polymyxin-B-associated acute kidney injury in 1-year mortality and renal function recovery. Int $\mathbf{J}$ Antimicrob Agents. 2018;52:86-9.

25. Landman D, Georgescu C, Martin DA, Quale J. Polymyxins revisited. Clin Microbiol Rev. 2008;21:449-65.

26. Kassamali Z, Danziger L. To B or not to B, that is the question: is it time to replace colistin with polymyxin B? Pharmacotherapy. 2015;35:1-27.

27. Falagas ME, Kasiakou SK. Toxicity of polymyxins: a systematic review of the evidence from old and recent studies. Crit Care. 2006;10:R27.

28. Vardakas KZ, Falagas ME. Colistin versus polymyxin B for the treatment of patients with multidrug-resistant Gram negative infections: a systematic review and meta-analysis. Int J Antimicrob Agents. 2017;49:233-8.

29. Justo JA, Bosso JA. Adverse reactions associated with systemic polymyxin therapy. Pharmacotherapy. 2005;35:28-33.

30. Oliveira MS, Prado GV, Costa SF, Grinbaum RS, Levin AS. Polymyxin B and colistimethate are comparable as to efficacy and renal toxicity. Diagn Microb Infect Dis. 2009;65:431-4.

31. Rigatto MH, Oliveira MS, Perdigão-Neto MT, Levin AS, Carrilho CM, Tanita MT, et al. Multicenter prospective cohort study of renal failure in patients treated with colistin versus polymyxin B. Antimicrob Agents Chemother. 2016;60:2443-9.

32. Kwa AL, Tam VH, Falagas ME. Polymyxins: a review of the current status including recent developments. Ann Acad Med Singap. 2008:37:870-83. 